\title{
Measurement of cross sections and couplings of the Higgs bo- son in fermionic decay modes with the ATLAS detector
}

\author{
Liaoshan Shi, ${ }^{1,2, a}$, on behalf of the ATLAS Collaboration \\ ${ }^{1}$ Institute of Physics, Academia Sinica, Taipei, Taiwan \\ ${ }^{2}$ School of Physics, Sun Yat-sen University, Guangzhou, China
}

\begin{abstract}
In this report, we present the latest ATLAS results on the measurement of the cross sections and couplings of the Higgs boson in the fermionic decay modes, $H \rightarrow$ $\mu^{+} \mu^{-}, H \rightarrow \tau^{+} \tau^{-}$and $H \rightarrow b \bar{b}$. The searches are performed with proton-proton collision data delivered by the Large Hadron Collider during Run 1 and the first two years of Run 2 at $\sqrt{s}=7,8$ and $13 \mathrm{TeV}$.
\end{abstract}

\section{Introduction}

In 2012, the ATLAS and CMS Collaborations observed a new particle with a mass of approximately $125 \mathrm{GeV}[1,2]$ in analysing the proton-proton $(p p)$ collision data delivered by the Large Hadron Collider (LHC) [3]. The properties of the discovered particle were found to be compatible with those of a Standard Model (SM) Higgs boson.

Precise measurements of the coupling of the Higgs boson to other SM particles can provide further insight into the nature of electroweak symmetry breaking. Many of those measurements rely predominantly on studies of the bosonic decay modes, $H \rightarrow \gamma \gamma, H \rightarrow Z Z^{*}$ and $H \rightarrow W W^{*}$. To study the Yukawa couplings between the Higgs boson and the fermions, and test the mass-coupling relation predicted by the SM, it is of particular importance to measure the direct coupling of the Higgs boson to fermions. The ATLAS experiment [4] has searched the Higgs boson in fermionic decay modes $H \rightarrow \mu^{+} \mu^{-}, H \rightarrow \tau^{+} \tau^{-}$and $H \rightarrow b \bar{b}$. The searches are done using three different main production modes: gluon fusion (ggF), vector boson fusion (VBF) and associated production with a vector boson $(V H)$. The ggF mode has the largest cross section among all the production modes. It is used to study the leptonic decay modes $H \rightarrow \mu^{+} \mu^{-}$and $H \rightarrow \tau^{+} \tau^{-}$with clean signatures but low branching ratios. The VBF production mode has the second-largest cross section. It is characterised by two forward VBF jets from light quarks, and a large rapidity gap with low hadronic activities due to no color connection between the Higgs boson and the VBF jets. The VBF mode is used in the studies of all the three fermionic decay modes. Finally, the $V H$ production mode targets a Higgs boson produced in association with a $W$ or $Z$ boson. The leptonic decay of the vector bosons leads to clean signatures that can be efficiently triggered on. The $V H$ mode is used in the studying of the $H \rightarrow \tau^{+} \tau^{-}$and $H \rightarrow b \bar{b}$ decay modes.

In this report, we present the latest ATLAS results in searching for the SM Higgs boson in $H \rightarrow$ $\mu^{+} \mu^{-}, H \rightarrow \tau^{+} \tau^{-}$and $H \rightarrow b \bar{b}$ decay modes using $p p$ collision data collected during LHC Run 1 and

\footnotetext{
a e-mail: Liaoshan.Shi@ cern.ch
} 
Run 2 data taking periods. The $H \rightarrow \mu^{+} \mu^{-}$search, performed with a Run 2 dataset at a centre-of-mass energy of $\sqrt{s}=13 \mathrm{TeV}$, is described in Sect. 2. The $H \rightarrow \tau^{+} \tau^{-}$searches, performed with Run 1 datasets at $\sqrt{s}=7$ and $8 \mathrm{TeV}$, are described in Sect. 3. The $H \rightarrow b \bar{b}$ searches, performed with Run 1 and Run 2 datasets at $\sqrt{s}=8$ and $13 \mathrm{TeV}$, are described in Sect. 4 .

\section{$2 H \rightarrow \mu^{+} \mu^{-}$}

The $H \rightarrow \mu^{+} \mu^{-}$decay is a clean final-state signature to measure the Higgs coupling to second generation fermions. The latest search is carried out using $p p$ collision data recorded in 2015 and 2016 at $\sqrt{s}=13 \mathrm{TeV}$, corresponding to an integrated luminosity of $36.1 \mathrm{fb}^{-1}$ [5].

This analysis targets both the $\mathrm{ggF}$ and the VBF signatures. The inclusive signal region is formed by selecting dimuon events with exactly two opposite-charge muons. The dominant irreducible background is the $Z / \gamma^{*} \rightarrow \mu \mu$ (Drell-Yan) process. Figure 1 (left) shows the dimuon invariant mass $m_{\mu \mu}$ distributions for data and simulated events in the inclusive signal region.

To enhance the analysis sensitivity, events in the inclusive signal region are classified into eight orthogonal categories. For events containing at least two jets, a Boosted Decision Tree (BDT) [6, 7] is trained to maximise the separation between the VBF signal and the total background. Two VBF categories, VBF tight and VBF loose, are defined from events with high BDT scores. The remaining events that are not selected for the VBF categories, including events with low BDT scores and events with less than two jets, all enter into the ggF categories. These events are further divided into six categories according to the transverse momentum of the dimuon system (low, medium and high $p_{\mathrm{T}}^{\mu \mu}$ categories) and the pseudorapidities of the muons (central and non-central categories).

A binned maximum-likelihood fit to the observed $m_{\mu \mu}$ distribution in the range $110-160 \mathrm{GeV}$ is performed simultaneously in all categories to determine the background and extract the signal strength $\mu$, which is defined as the measured signal yield normalised to the SM prediction. No significant excess is observed in data. In figure 1 (right), the observed $m_{\mu \mu}$ distribution in the most sensitive category, the VBF tight category, is compared to the background-only fit. The measured signal strength is $\mu=-0.1 \pm 1.5$. An upper limit on $\mu$ is computed using a modified frequentist $\mathrm{CL}_{s}$ method $[8,9]$. The observed (expected) upper limit on $\mu$ at the 95\% confidence level (CL) is found to be 3.0 (3.1), driven by the data statistical uncertainty. When combined with the ATLAS Run 1 data, the measured signal strength is found to be $\mu=-0.1 \pm 1.4$, and the observed (expected) upper limit is 2.8 (2.9) at the $95 \%$ CL.

\section{$3 H \rightarrow \tau^{+} \tau^{-}$}

The $H \rightarrow \tau^{+} \tau^{-}$decay has been probed with full Run $1 \mathrm{pp}$ collision data recorded at centre-ofmass energies of $\sqrt{s}=7 \mathrm{TeV}$ and $8 \mathrm{TeV}$. The datasets correspond to integrated luminosities of $4.5 \mathrm{fb}^{-1}$ and $20.3 \mathrm{fb}^{-1}$, respectively [10]. All combinations of leptonic and hadronic tau decays that are considered in this analysis, are explored by three analysis channels: $\tau_{\text {lep }} \tau_{\text {lep }}, \tau_{\text {lep }} \tau_{\text {had }}$ and $\tau_{\text {had }} \tau_{\text {had }}$. To exploit signal-sensitive event topologies, two analysis categories are defined in an exclusive way: The VBF category targets VBF events by requiring the presence of two high- $p_{\mathrm{T}}$ jets with a large pseudorapidity separation. The boosted category targets events with a boosted Higgs boson produced via $\mathrm{ggF}$ by requiring the Higgs boson to have large transverse momentum. Small contributions from the $V H$ production are also considered as signal. The main background processes include $Z / \gamma^{*} \rightarrow \tau \tau$, top-quark productions, and misidentified taus. The $Z / \gamma^{*} \rightarrow \tau \tau$ background is modelled using an embedding technique which uses $Z / \gamma^{*} \rightarrow \mu \mu$ events from data.

The ditau invariant mass $m_{\tau \tau}^{\mathrm{MMC}}$ is reconstructed using the missing mass calculator (MMC) method by solving an under-constrained system of equations for six to eight unknowns, depending on the 

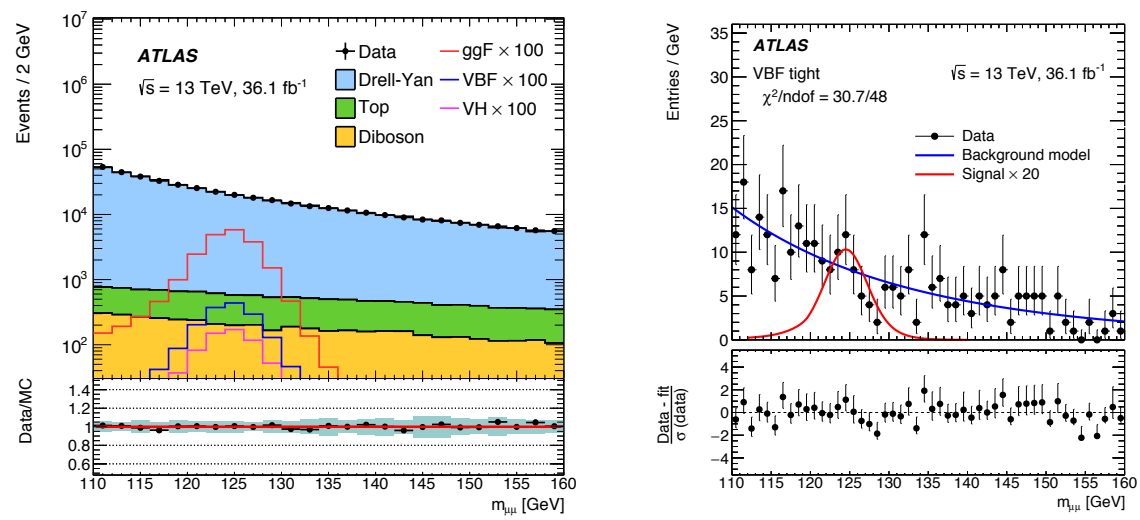

Figure 1. Observed and simulated $m_{\mu \mu}$ distributions in the inclusive signal region (left), and background-only fit to the observed $m_{\mu \mu}$ distribution in the VBF tight category (right) in the $H \rightarrow \mu^{+} \mu^{-}$analysis [5].

number of neutrinos in the $\tau \tau$ final state. Figure 2 (left) shows the reconstructed $m_{\tau \tau}^{\mathrm{MMC}}$ distribution for all channels summed, wighted by $\ln (1+S / B)$, where $S$ and $B$ denote the signal and background yields, respectively. Boosted decision trees are used in each category as the final discriminant to extract the Higgs boson signal from the large number of background events. A maximumlikelihood fit is performed on all categories simultaneously to extract the signal strength. It is measured to be $\mu=1.43_{-0.26}^{+0.27}$ (stat.) ${ }_{-0.25}^{+0.32}$ (syst.) \pm 0.09 (theory syst.), which corresponds to a deviation from the background-only hypothesis of $4.5 \sigma$, compared to an expected significance of $3.4 \sigma$. The signal strengths of different Higgs boson production processes are also determined by fitting the data with two signal strengths to separate the vector-boson-mediated VBF and $V H$ processes from gluonmediated ggF processes. The two-dimensional $68 \%$ and $95 \% \mathrm{CL}$ contours in the plane of $\mu_{\mathrm{ggF}}^{\tau \tau}$ and $\mu_{\mathrm{VBF}+V H}^{\tau \tau}$ are shown in figure 2 (right).

When combined with the CMS Run 1 data, the excess corresponds to a discovery significance of $5.5 \sigma$, to be compared with an expectation of $5.0 \sigma$ [11].

Another analysis targeting specifically the $V H$ production mode is performed with $20.3 \mathrm{fb}^{-1}$ data at $\sqrt{s}=8 \mathrm{TeV}$, and an upper limit on the signal strength is set at 95\% CL. The observed (expected) limit is 5.6 (3.5). The measured signal strength is $\mu=2.3 \pm 1.6$ [12].

\section{$4 H \rightarrow b \bar{b}$}

The $H \rightarrow b \bar{b}$ decay is expected to have the largest branching ratio of $58 \%$ for a $125 \mathrm{GeV}$ SM Higgs boson [13]. Measurements in this decay mode are crucial to constrain the overall Higgs boson decay width. However, an inclusive search in this decay mode is extremely challenging due to the overwhelming background from multi-jet production. Several ATLAS searches have been carried out targeting the $\mathrm{VBF}$ and $V H$ signatures.

\section{1 $H \rightarrow b \bar{b}$ in vector boson fusion production}

The VBF $H \rightarrow b \bar{b}$ search is performed using Run $1 p p$ collision data at a centre-of-mass energy $\sqrt{s}=8 \mathrm{TeV}$, corresponding to an integrated luminosity of $20.2 \mathrm{fb}^{-1}$ [14]. Although the search targets 

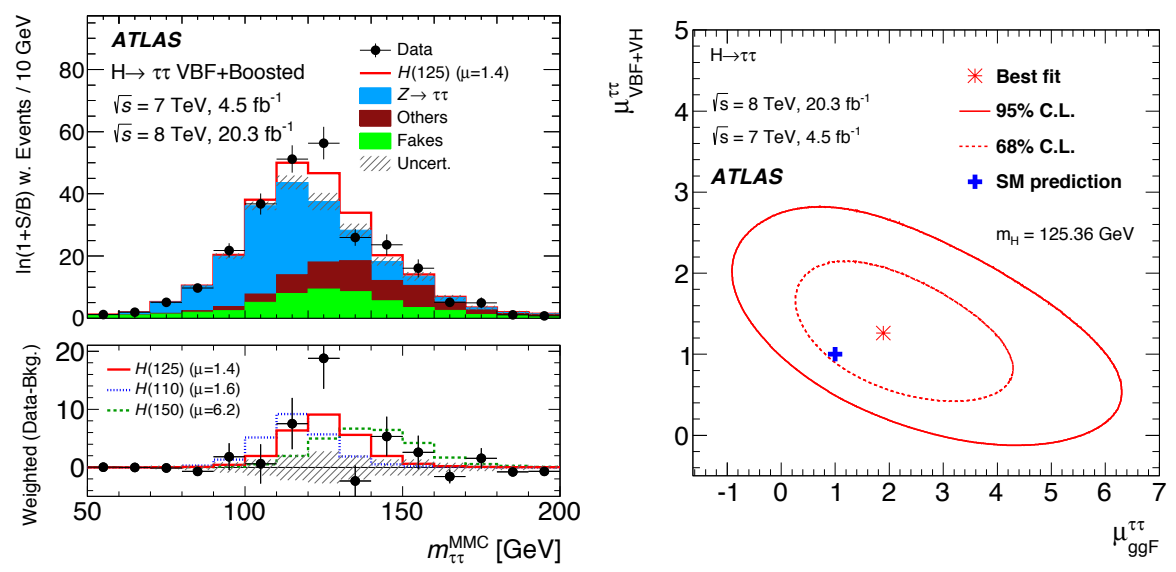

Figure 2. Left: Distribution of the reconstructed invariant $\tau \tau$ mass $m_{\tau \tau}^{\mathrm{MMC}}$, where events are weighted by $\ln (1+$ $S / B$ ) for all channels. Right: Likelihood contours in the plane of two signal strength parameters $\mu_{\mathrm{ggF}}^{\tau \tau}$ and $\mu_{\mathrm{VBF}+V H}^{\tau \tau}$ in the $H \rightarrow \tau^{+} \tau^{-}$analysis [10].

the VBF signature, any possible contributions from the ggF and $V H$ productions are also considered as signal.

Signal events are selected by requiring four energetic jets in the final state: two light-quark jets from VBF process at a small angle with respect to the beam line, and two $b$-jets from the Higgs boson decay in a more central region. This is denoted as the $b \bar{b} j j$ final state. The dominant background is from non-resonant multi-jet production. The non-resonant background is modelled using a data-driven method by fitting data in the sidebands of the $m_{b b}$ to an analytic function, which is then interpolated to the signal region. To improve the sensitivity, a BDT is used to exploit the characteristics of the VBF topology and the properties of jets. The input variables of the BDT are chosen to maximise the separation between signal events and background events while keeping them as uncorrelated as possible with $m_{b b}$, to ensure that the sideband regions provide a good description of the non-resonant background in the signal region. The BDT response of data and the simulated events are shown in figure 3 (left). Events are categorised into four categories based on the BDT response. The boundaries of the categories are optimised by minimising the relative statistical uncertainties.

The signal is extracted using a binned likelihood fit to the $m_{b b}$ distributions in the range $70<m_{b b}<$ $300 \mathrm{GeV}$. The fit is performed simultaneously in all BDT categories. The Higgs signal strength is measured to be $\mu=-0.8 \pm 2.3$. The observed (expected) upper limit on the signal strength at $95 \% \mathrm{CL}$ is 4.4 (5.4). The analysis sensitivity is limited by the uncertainty of the non-resonant background, which is dominated by the statistical uncertainty in the data sidebands used to determine the background.

\section{2 $H \rightarrow b \bar{b}$ in vector boson fusion production in association with a photon}

The inclusive VBF $H \rightarrow b \bar{b}$ analysis is challenging due to very large multi-jet background contribution and the difficulty in triggering on low $p_{\mathrm{T}} b$-jets. ATLAS has explored a new channel for the first time by requiring an additional photon in the final state, denoted as the $b \bar{b} \gamma j j$ final state. The photon can be radiated by the initial or final state quark, or by the intermediate $W$ boson. A typical Feynman diagram for the signal process is shown in figure 4 (left). The first result of the search for the VBF 

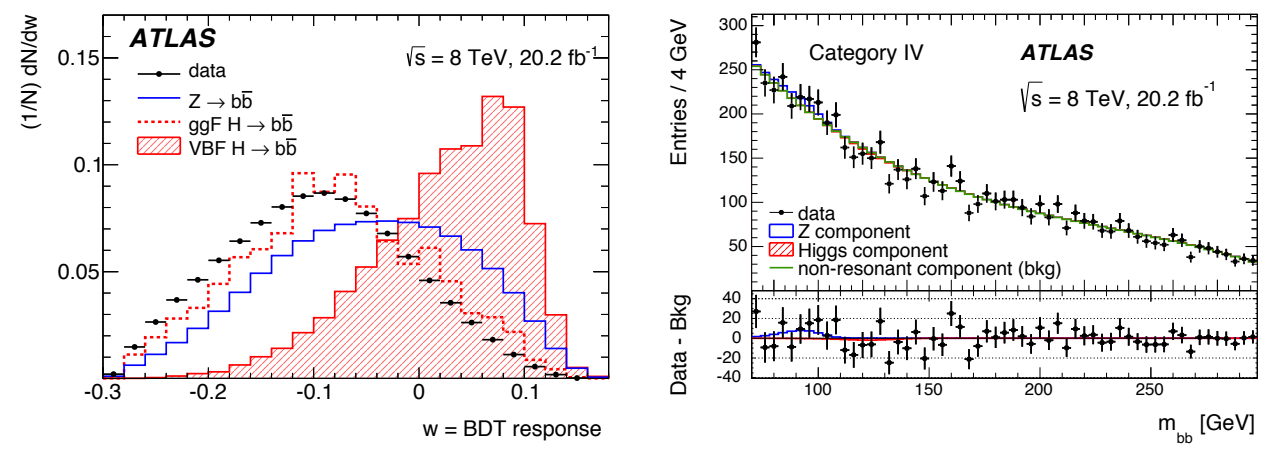

Figure 3. Distributions of the BDT response to the data and to the simulated events (left), ant results of the profile-likelihood fit to the $m_{b b}$ distribution in the most sensitive BDT category (right) in the VBF $H \rightarrow b \bar{b}$ analysis [14].

Higgs boson production in the $b \bar{b} \gamma j j$ final state is obtained by analysing a set of $12.6 \mathrm{fb}^{-1} p p$ collision data collected with the ATLAS detector at $\sqrt{s}=13 \mathrm{TeV}$ [15].

Although the signal rate in the $b \bar{b} \gamma j j$ final state is reduced by approximately two orders of magnitude comparing to the inclusive $b \bar{b} j j$ final state, the signal-to-background ratio is still enhanced due to stronger reduction of the non-resonant background by destructive interference between initial and final state radiation diagrams and suppression in the gluon-induced processes. The high- $p_{\mathrm{T}}$ photon also provides a clean signature for efficient triggering. A dedicated trigger targeting the $b \bar{b} \gamma j j$ final state is used to select data events.

A similar analysis strategy as the inclusive VBF $H \rightarrow b \bar{b}$ analysis is used to analyse the selected events. Events are categorised into three BDT regions (low, medium and high BDT regions). The signal extraction is performed by fitting the $m_{b b}$ distribution simultaneously in all BDT regions. Benefit from the dedicated trigger with lower $b$-jet $p_{\mathrm{T}}$ threshold, the lower $m_{b b}$ boundary in the fit is extended down to $50 \mathrm{GeV}$, providing a better estimation of the data-driven background description. The fitted backgrounds in the high BDT region are compared to data in figure 4 (right). The measured signal strength is $\mu=-3.9_{-2.7}^{+2.8}$. The observed (expected) upper limit at $95 \% \mathrm{CL}$ is 4.0 (6.0). The analysis sensitivity is limited by data statistical uncertainty.

\section{3 $H \rightarrow b \bar{b}$ in associated production with a vector boson}

The most sensitive production mode for probing $H \rightarrow b \bar{b}$ decay is the associated production with a $W$ or $Z$ boson. The leptonic decays of the vector boson lead to clean signatures for triggering while rejecting most of the multi-jet background. The latest search in the $V H$ mode is performed with a Run 2 dataset collected at a centre-of-mass energy of $\sqrt{s}=13 \mathrm{TeV}$, corresponding to an integrated luminosity of $36.1 \mathrm{fb}^{-1}$ [16].

The analysis is split into three channels, denoted by the number of charged leptons in the final states (0-, 1- and 2-lepton channels), exploiting different decay modes of the vector boson: $Z H \rightarrow$ $v v b \bar{b}, W H \rightarrow \ell v b \bar{b}$ and $Z H \rightarrow \ell \ell b \bar{b}$. Events are further split into regions based on the number of jets and the $p_{\mathrm{T}}$ of the vector bosons. The main background processes include contributions of multi-jet production from strong interactions, vector boson production with jets and top-quark productions. Figure 5 shows the reconstructed vector boson mass or transverse mass distributions with simulated background compositions in each channel, and the total background comparing to data. 

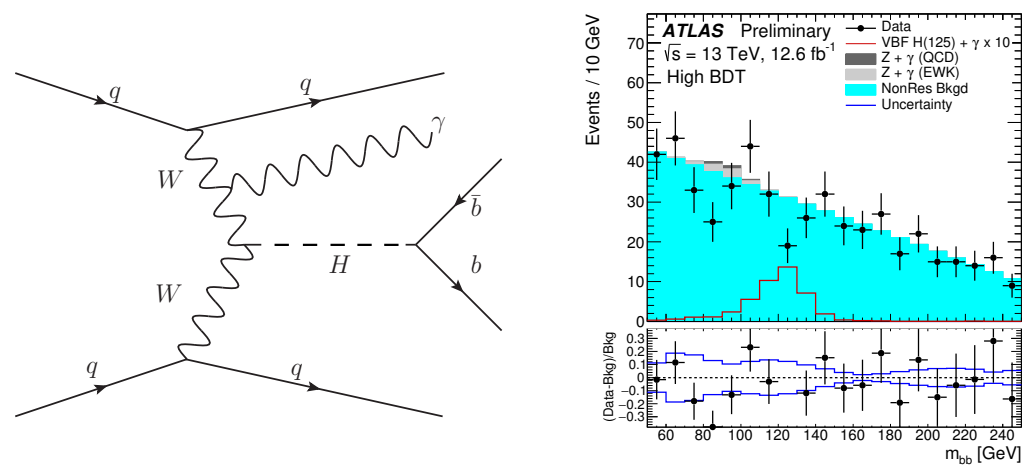

Figure 4. Representative leading-order Feynman diagrams for Higgs boson signal production (left), and the invariant mass distribution for the most sensitive $\mathrm{BDT}$ region (right) in the VBF $H \rightarrow b \bar{b}+\gamma$ analysis [15].

To maximise the sensitivity to the Higgs boson signal, a multivariate analysis (MVA) is setup making use of BDTs. In each signal regions, a BDT is constructed, trained and evaluated separately with a set of observables encoding information on event kinematics and topology. The BDT discriminant from each signal region is used as the main observable in a global likelihood fit to extract the Higgs boson signal strength. The observed signal strength for all channels combined is $\mu=1.20_{-0.23}^{+0.24}$ (stat. $)_{-0.28}^{+0.34}$ (syst.), corresponding to an excess with a significance of $3.5 \sigma$, comparable to the SM expectation of 3.0 $\sigma$. Figure 6 (left) shows the data, background and signal yields where final-discriminant bins in all regions are combined into bins of $\log (S / B)$ with $S$ and $B$ referring to the fitted signal and background yields, respectively. When combined with the ATLAS Run 1 data, the measured signal strength is $\mu=0.9 \pm 1.8$ (stat. ${ }_{-0.19}^{+0.21}$ (syst.), corresponding to an excess with a significance of $3.6 \sigma$, to be compared to an expectation of $4.0 \sigma$.
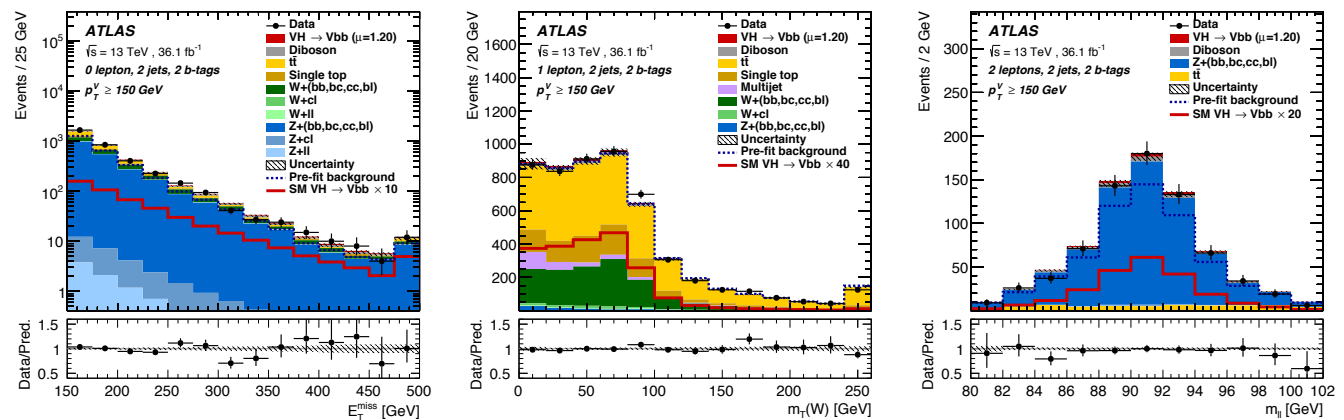

Figure 5. The post-fit distributions for $E_{\mathrm{T}}^{\mathrm{miss}}$ (left), $m_{\mathrm{T}}^{W}$ (middle) and $m_{\ell \ell}$ (right) in the 0-lepton, 1-lepton and 2-lepton channels for 2-jet, 2-b-tag events in the high $p_{\mathrm{T}}^{V}$ region of the $V H H \rightarrow b \bar{b}$ analysis [16].

The results of the MVA analysis are cross-checked by a diboson analysis and a dijet-mass analysis. In the diboson analysis, the $Z$ boson signal strength instead of the Higgs boson signal strength is extracted using the same method to validate the overall method of the MVA analysis. The measured signal strength is $\mu_{V Z}=1.11_{-0.11}^{+0.12}$ (stat. ${ }_{-0.19}^{+0.22}$ (syst.), in good agreement with the SM prediction. The 
data, background and signal yields of the diboson analysis is shown in figure 6 (middle). In the dijet-mass analysis, the BDT discriminant is replaced by the $m_{b b}$ variable as the main observable in the global fit. The measured signal strength is $\mu=1.30_{-0.27}^{+0.28}$ (stat. $)_{-0.29}^{+0.37}$ (stat.), in good agreement with the MVA result. The $m_{b b}$ distribution for all regions summed, weighted by their respective $S / B$ after subtraction of all backgrounds except for the $W Z$ and $Z Z$ diboson processes, is shown in figure 6 (right). The data and the sum of expected signal and background yields are in good agreement.
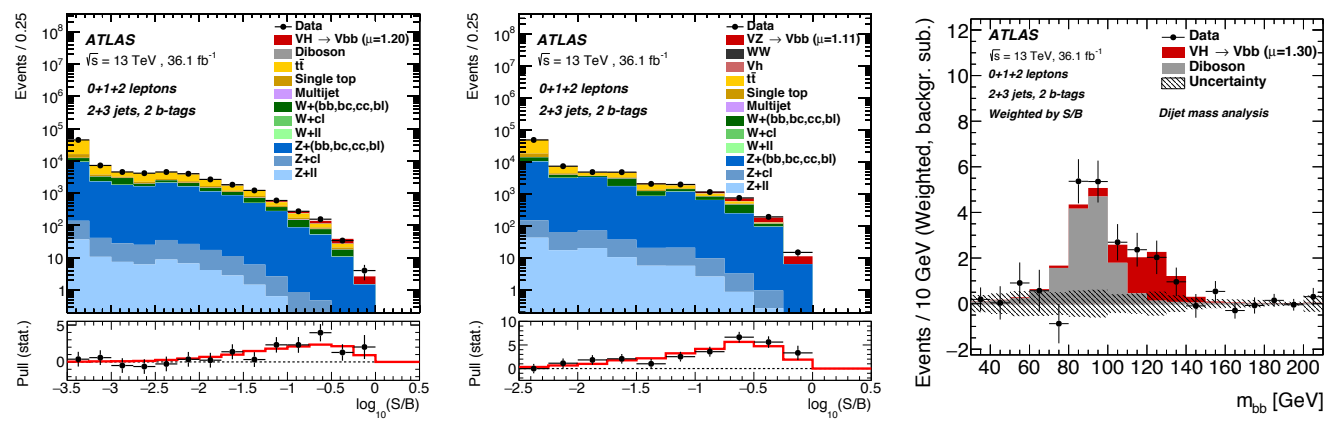

Figure 6. Data, signal and background yields from the MVA (left), diboson (middle) and dijet-mass (right) analyses in the $V H H \rightarrow b \bar{b}$ search [16].

\section{Couclusion}

Measurement of the couplings of the Higgs boson to fermions is essential to understand the properties of the Higgs boson and test the Standard Model predictions. ATLAS is actively exploring the direct couplings of the Higgs boson to fermions in fermionic decay modes of the Higgs boson. Latest results in the $H \rightarrow \mu^{+} \mu^{-}, H \rightarrow \tau^{+} \tau^{-}$and $H \rightarrow b \bar{b}$ searches are presented, and evidence of the Higgs boson decaying to tau leptons and $b$-quarks has been observed. ATLAS also explored a new VBF $+\gamma$ channel to access the $H \rightarrow b \bar{b}$ decay for the first time. These analyses anticipate updated results with more data in the near future.

\section{References}

[1] ATLAS Collaboration, Phys. Lett. B716, 1 (2012), 1207.7214

[2] CMS Collaboration, Phys. Lett. B716, 30 (2012), 1207.7235

[3] L. Evans, P. Bryant, Journal of Instrumentation 3, S08001 (2008)

[4] ATLAS Collaboration, Journal of Instrumentation 3, S08003 (2008)

[5] ATLAS Collaboration, Phys. Rev. Lett. 119, 051802 (2017), 1705.04582

[6] L. Breiman et al., Classification and Regression Trees (Wadsworth and Brooks, Monterey, CA, 1984)

[7] B.P. Roe et al., Nucl. Instrum. Meth. A543, 577 (2005), physics/0408124

[8] G. Cowan, K. Cranmer, E. Gross, O. Vitells, Eur. Phys. J. C71, 1554 (2011), [Erratum: Eur. Phys. J.C73,2501(2013)], 1007.1727

[9] A.L. Read, Journal of Physics G: Nuclear and Particle Physics 28, 2693 (2002)

[10] ATLAS Collaboration, JHEP 04, 117 (2015), 1501 . 04943 
[11] ATLAS and CMS Collaborations, JHEP 08, 045 (2016), 1606.02266

[12] ATLAS Collaboration, Phys. Rev. D93, 092005 (2016), 1511.08352

[13] A. Djouadi, J. Kalinowski, M. Spira, Comput. Phys. Commun. 108, 56 (1998), hep-ph/9704448

[14] ATLAS Collaboration, JHEP 11, 112 (2016), 1606.02181

[15] ATLAS Collaboration, Tech. Rep. ATLAS-CONF-2016-063, CERN, Geneva (2016), https: //cds. cern. ch/record/2206201

[16] ATLAS Collaboration (2017), 1708.03299 